\title{
Colangiocarcinoma hepático en un felino y hallazgos anatomopatólogicos, y clínicos compatibles con peritonitis infecciosa felina
}

\section{Hepatic cholangiocarcinoma in a feline and anatomopathologic and clinical findings, compatible with Feline Infectious Peritonitis}

\author{
Julieta Ochoa A, ${ }^{1}$ M.Sc, AI Roque, ${ }^{1}$ M.Sc, JA Daza $H,{ }^{1}$ MVZ
}

\begin{abstract}
${ }^{1}$ Universidad de los Llanos, Facultad de Ciencias Agropecuarias y Recursos Naturales, Escuela de Medicina Veterinaria y Zootecnia, Villavicencio, Meta, Colombia *Correspondencia: julietaeochoa@ yahoo.es
\end{abstract}

Recibido: Marzo de 2010; Aceptado: Diciembre de 2011.

\begin{abstract}
RESUMEN
Se presentan los hallazgos patomorfológicos de colangiocarcinoma hepático (CGC) con efusión peritoneal en un gato doméstico geronte y se relaciona con la sintomatología clínica y los hallazgos anatomopatológicos compatibles con la peritonitis infecciosa felina (PIF). Se atendió en la clínica veterinaria de la Universidad de los Llanos un paciente felino de 8 años de edad, criollo, con aumento de la silueta abdominal y depresión. Clínicamente se encontró deshidratación del 7\%, hipotermia $\left(37.2^{\circ} \mathrm{C}\right)$, mucosas pálidas, disnea y hepatomegalia. Mediante abdominocentesis se obtuvo un exudado no séptico y un cuadro citológico compatible con CGC con células columnares bajas dispuestas en panal de abeja, presentando anisocariosis, hipercromacia moderada, citoplasmas claros y vacuolados acompañados de un fondo sucio inflamatorio y hemorrágico con detritos celulares. En el análisis radiográfico se observó hepatomegalia con un patrón de punteado característico de formación neoplásica y ascitis. A la necropsia se presentó hemotórax e hidropericardio, ascitis y nódulos neoplásicos blancos duros al corte en el $45 \%$ del parénquima hepático y un patrón nodular sobre la superficie visceral del estomago, intestinos delgado, grueso y mesenterio. Las muestras se fijaron en formalina buferada al $10 \%$, se procesaron mediante métodos rutinarios para microscopía óptica. Por histopatología se diagnosticó CGC moderadamente diferenciado con intensa desmoplasia. Citológica e histopatológicamente se evidenció un CGC hepático con efusión peritoneal que se relacionó con la sintomatología clínica y los hallazgos anatomopatológicos compatibles con la PIF.
\end{abstract}

Palabras clave: Colangiocarcinoma, deshidratación, duto biliar, gatos (Fuentes: DeCS, CAB). 


\section{ABSTRACT}

Path morphological findings are presented regarding hepatic cholangiocarcinoma (CGC) with peritoneal effusion in a elderly domestic cat; its relation with clinical symptoms and pathological findings consistent with feline infectious peritonitis (FIP). The veterinary clinic of the Universidad de los Llanos dealt with a feline patient 8 years old, criollo, with increased abdominal silhouette and depression. $7 \%$ dehydration, hypothermia $\left(37.2^{\circ} \mathrm{C}\right)$, pale mucous membranes, dyspnea and hepatomegaly were determined clinically. A performed abdominocentesis presented nonseptic exudate and a cytological symptom consistent with CGC with low columnar cells arranged in a honeycomb structure, presenting anisokaryosis, moderate hipercromacia, clear cytoplasm and accompanied by a dirty inflammatory and hemorrhagic background with cellular debris. The radiographic analysis showed hepatomegaly with a dotted pattern characteristic of neoplastic and ascite formations. A necropsy showed hemothorax and hydropericardium, ascites and white solid neoplastic nodules in $45 \%$ of the liver parenchyma and nodular pattern on the visceral surface of the stomach, small, large and mesentery intestine. Samples were placed in $10 \%$ buffered formalin, processed by routine methods for optic microscopy. In relation to histopathology moderately differentiated CGC was diagnosed with intense desmoplasia. A hepatic CGC was evidenced Cytologicly as well as histopathológicly with peritoneal effusion that was associated with clinical symptoms and anatomopathological findings consistent with FIP.

Key words: Cholangiocarcinoma, cats, dehydration, bile ducts (Sources: DeCS, CAB).

\section{INTRODUCCIÓN}

Los tumores hepatobiliares en gatos ocurren con muy baja frecuencia, entre esos esta el colangiocarcinoma (CGC) (1) o carcinoma de los ductos biliares, sin embargo el ha sido reportado en gatos viejos y en ellos es la neoplasia hepática más frecuente (2), no se conoce que tenga predilección por raza $(2,3)$. La prevalencia varía desde 1.5 a $2.3 \%$ de las neoplasias felinas y en un estudio retrospectivo de 47 neoplasias de los sistemas hepático y biliar de 47 gatos llevados al centro médico de los animales durante un período de 10 años (1980 a 1989), se reveló que al examen histológico de las muestras tomadas en la autopsia el $87 \%$ (41/47) de las neoplasias hepáticas fueron epiteliales y el $13 \%(6 / 47)$ no epiteliales (4). De los tumores epiteliales, el $53 \%$ eran de orígen biliar intrahepático (25/47), el $19 \%$ eran de orígen hepatocelular (9/47), un $11 \%$ (5/47) de los conductos biliares extrahepáticos y un $4 \%(2 / 47)$ fueron adenocarcinomas de la vesícula biliar (4), permitiendo concluir que las neoplasias epiteliales y aquellas originadas del sistema biliar fueron más comunes que las no epiteliales y que los carcinomas hepatocelulares respectivamente (4).

Las manifestaciones clínicas de las neoplasias hepatobiliares en felinos son inespecíficas como letargia, vómito, pérdida de peso, disnea, polidipsia y poliurea $(5,6)$, ascitis, hipoalbuminemia y hepatomegalia (2) su diagnóstico raramente es llevado a cabo en vivo y en muchos casos el diagnóstico definitivo se dá después de la necropsia (1). Macroscópicamente pueden tener apariencia masiva o multinodular $(2,5)$ inclusive pueden reemplazar lóbulos completos y diseminarse a los adyacentes. En los CGC bien diferenciados histopatológicamente las células semejan el epitelio biliar, son arregladas en forma tubular o acinar (2), los menos diferenciados tienen forma acinar y los pobremente diferenciados son compuestos de paquetes, islas o cordones celulares donde se pueden presentar áreas con diferenciación escamosas $(2,5)$ y una intensa desmoplasia, con un estroma denso de colágeno que separa los elementos glandulares (5). El pronóstico es pobre debido a su alto poder metastásico $(1,6)$.

La Peritonitis Infecciosa Felina (PIF) es la más importante enfermedad viral letal en felinos causada por un coronavirus, afecta a felinos domésticos y salvajes (7-9); se presenta en formas: efusiva o exudativa, con peritonitis (ascitis o pleuritis y serositis fibrosa), no efusiva o no exudativa con lesiones piogranulomatosas en órganos parenquimatosos, sistema nervioso central, ojos y finalmente la mixta (10). Aproximadamente el $80 \%$ de todos los gatos son infectados con el agente que causa la enteritis y de éstos el 5 al 12\% desarrollan los signos clínicos de PIF $(11,12)$, con mayor incidencia en los gatos de entre 6 meses y 5 años de edad, y la mayoría de los casos ocurre en los gatos $\leq 1$ año de edad (8), aunque también ha sido observada en animales de 17 años de edad enteros y no en castrados (13); según un estudio retrospectivo 
entre 1997-2000 la mayor incidencia de PIF fue en gatos machos que en hembras, siendo $62.4 \%$ y $37.6 \%$ respectivamente (8).

Internacionalmente han sido reportados muchos estudios sobre colangiocarcinomas en humanos y en varios animales, principalmente en perros, mientras que estudios sobre evaluación morfológica e histopatológica de CGC hepático en gatos son extremadamente raros $(2,4)$. Este reporte tuvo como objetivo presentar un caso de colangiocarcinoma hepático en un gato doméstico geronte, donde se describen los hallazgos patomorfológicos de CGC hepático con efusión peritoneal y se relaciona con la sintomatología clínica y los hallazgos anatomopatologicos compatibles con la PIF.

\section{HALLAZGOS CLÍNICOS}

Se atendio en la clínica de la Universidad de los Llanos un paciente felino de 8 años de edad y raza criolla, cuyo motivo de consulta fue decaimiento, aumento de la silueta abdominal, pérdida de la condición corporal y dificultad respiratoria. Al exámen clínico se encontró deshidratación del 7\%, hipotermia, mucosas pálidas, depósitos queratínicos sobre el endotelio corneal en el ojo derecho, bradicardia, pulso débil, disnea y hepatomegalia con parénquima lobulado (Figura 1).
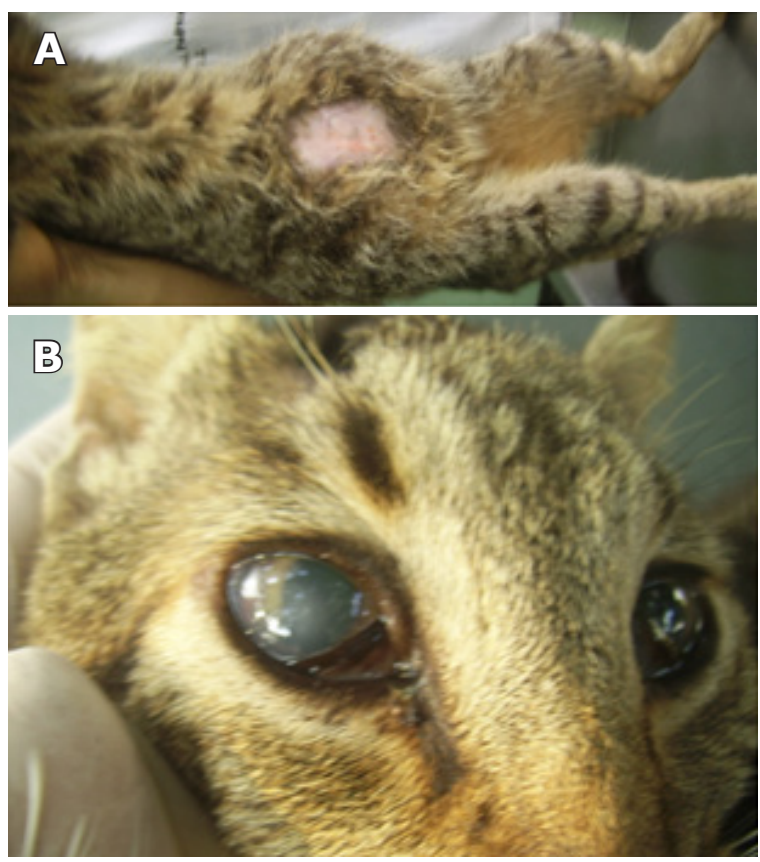

Figura 1. Felino doméstico con aumento de la silueta abdominal y depósitos queratínicos en el endotelio corneal derecho, respectivamente $(A, B)$.

\section{PRUEBAS PARACLINICAS}

Hemograma. Mostró linfopenia absoluta y neutrofilia absoluta y relativa (Tabla 1 ).

Tabla 1. Hemograma del paciente

\begin{tabular}{lll}
\hline \multicolumn{1}{c}{ ITEM } & \multicolumn{1}{c}{ HEMOGRAMA } & \multicolumn{1}{c}{ VALOR } \\
\hline Rto Leucocitario & $15.050 \times \mathrm{mm} 3$ & \multicolumn{1}{c}{$\begin{array}{c}\text { VALORES } \\
\text { NORMALES }\end{array}$} \\
Hematocrito & $37 \%$ & $487-20.100$ \\
Rto Glóbulos Bojos & $7.4 \times 106 \times \mathrm{mm} 3$ & $29.3-49.8 \%$ \\
VCM & $48 \mathrm{FI}$ & $41.9-51.8$ \\
Rto Plaquetas & $402.100 \times \mathrm{mm} 3$ & $26.000-470.000$ \\
Neutrofilos & $95 \%$ & $60-77 \%$ \\
Linfocitos & $5 \%$ & $15-35 \%$ \\
Neutrofilos & 14.297 & $2500-12.500$ \\
Linfocitos & 752.5 & $1500-7000$ \\
\hline
\end{tabular}

http://www.labdiagnotest.com/normales.php

Abdominocentesis. El líquido peritoneal se clasificó como un exudado no séptico (Tabla 2). A la lectura citológica se observó gran cantidad de material epitelial que corresponde a células columnares bajas dispuestas en panal de abeja presentando anisocariosis, moderada hipercromacia, citoplasmas claros y vacuolados acompañado de un fondo sucio inflamatorio

Tabla 2. Liquido peritoneal del paciente

\begin{tabular}{ll}
\multicolumn{2}{c}{ LIQUIDO PERITONEAL } \\
ITEM & \multicolumn{1}{c}{ VALOR } \\
\hline COLOR & ROJO \\
DENSIDAD & 1030 \\
ASPECTO & TURBIO \\
PH & 7.0 \\
PROTEINA & $7 \mathrm{gr} / \mathrm{dl}$ \\
RTO LEUCOCITARIO & $16.100 \times \mathrm{mm} 3$ \\
NEUTROFILOS & $65 \%$ \\
LINFOCITOS & $35 \%$ \\
RTO GLOBULOS ROJOS & $53.600 \times \mathrm{mm} 3$ \\
FRESCOS & $20 \%$ \\
CRENADOS & $80 \%$ \\
\hline
\end{tabular}

y hemorrágico con detritos celulares, cuadro citológico compatible con colangiocarcinoma (Figura $2 \mathrm{~A}$ y $2 \mathrm{~B}$ ).

Rayos x. En el análisis radiográfico se observó hepatomegalia con un patrón de punteado característico de formación neoplásica y ascitis (Figura 3). 

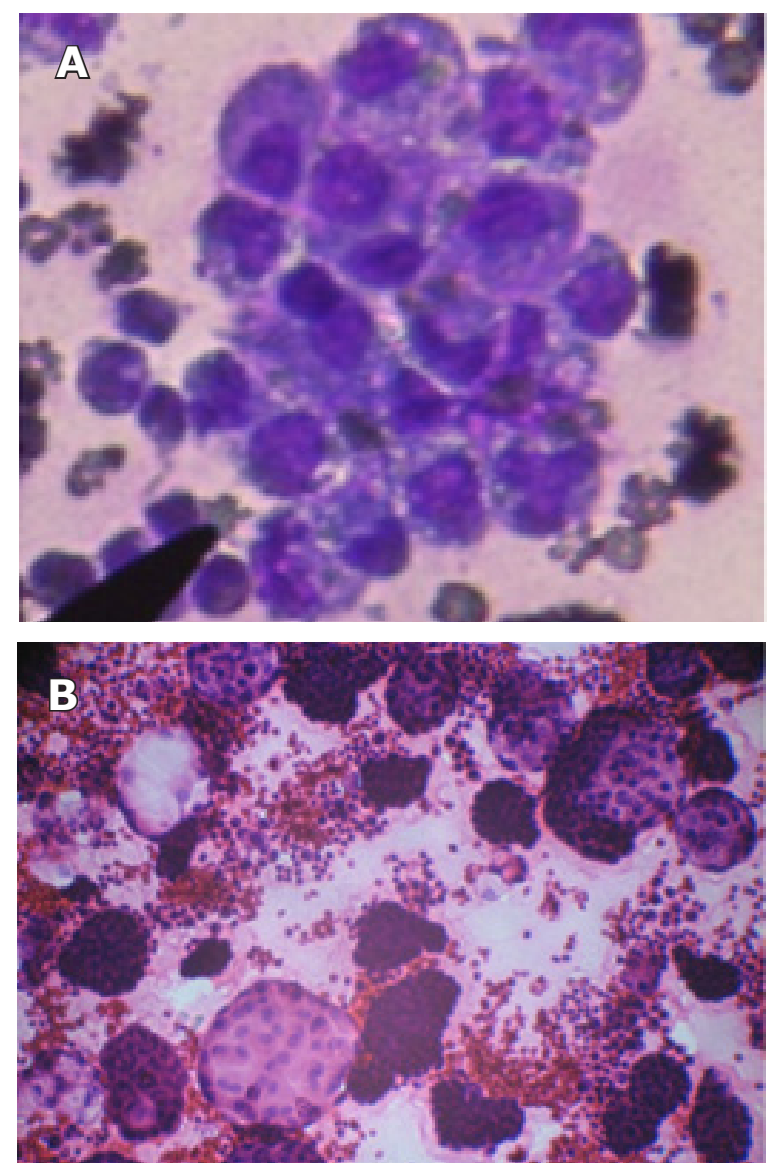

Figura 2. Células columnares bajas dispuestas en panal de abeja presentando anisocariosis, moderada hipercromacia, citoplasmas claros y vacuolados acompañado de un fondo sucio inflamatorio y hemorrágico con detritos celulares, cuadro citológico compatible con colangiocarcinoma (A, B).

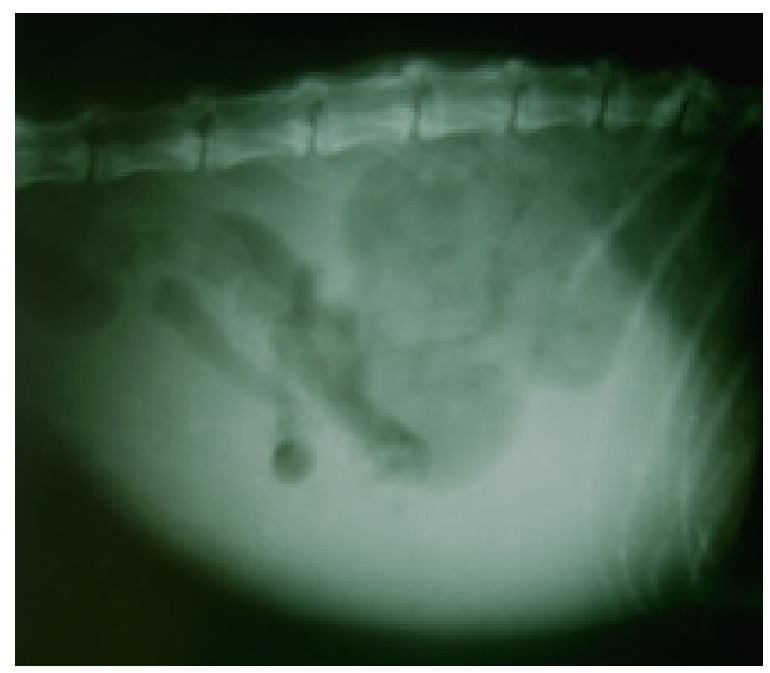

Figura 3. Hepatomegalia con patrón punteado en el hígado y ascitis.
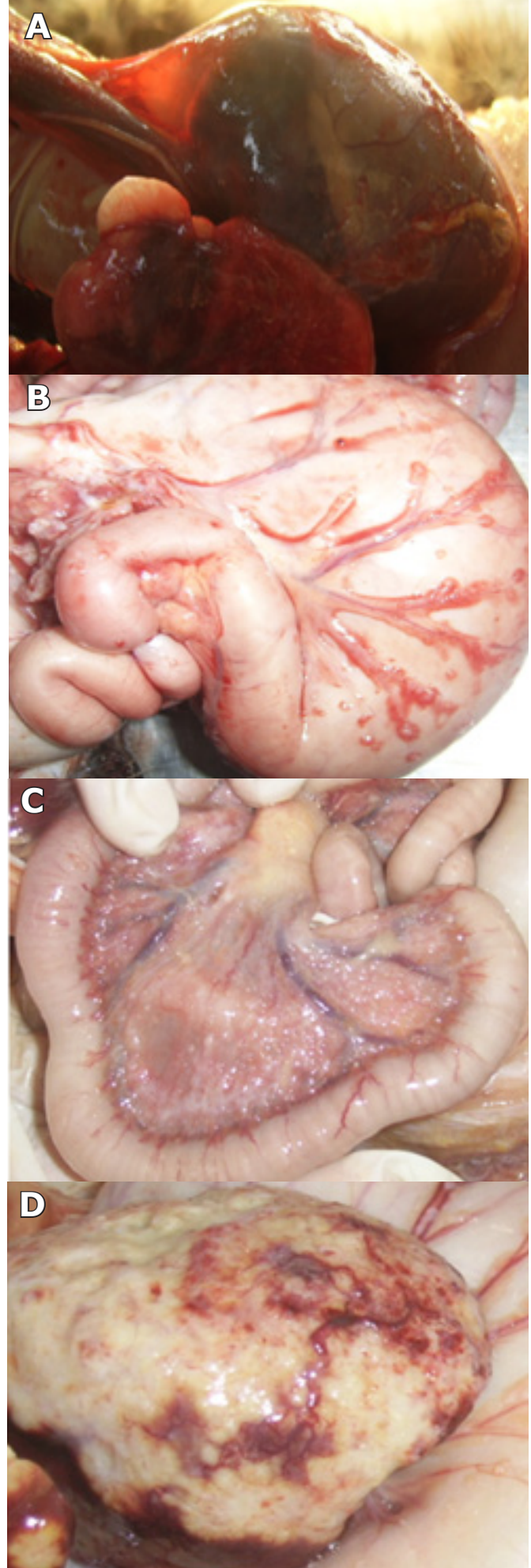

Figura 4. Hallazgos macroscópicos en el felino. Hidropericardio, (A). Deposiciones focales de exudado fibrinoso sobre la superficie visceral en estómago y peritoneo dando apariencia granular (B - C) así mismo nódulos neoplásicos hepáticos de consistencia dura al corte y coloración blanquecina en el $45 \%$ del parénquima (D). 


\section{HALLAZGOS MACROSCOPICOS}

Luego de ser autorizada la eutanasia se realizó la necropsia, encontrándose un animal con condición corporal $2 / 5$, pelo opaco e hirsuto y silueta abdominal aumentada de tamaño. Depósitos queratínicos sobre el endotelio corneal en el ojo derecho (Figura 1B), membranas mucosas pálidas, en la cavidad torácica se encontró hemotórax e hidropericardio (Figura $4 A$ ), en la cavidad abdominal se observó ascitis y deposiciones focales de exudado fibrinoso sobre la superficie visceral en estómago (Figura 4B) y superficie peritoneal (Figura 4C) dando apariencia granular, así mismo en el $45 \%$ del parénquima hepático se presentaron nódulos de consistencia dura al corte y de coloración blanquecina (Figura 4D), se tomaron muestras para histopatología, se fijaron en formalina buferada al $10 \%$ y los tejidos se procesaron por métodos rutinarios para microscopia óptica, fueron coloreados con Hematoxilina- eosina.

Morfológicamente los cambios anatomopatológicos observados como derrames abdominales, torácicos, plétora sanguínea en el epicardio, sugieren la presencia de un proceso viral concomitante con la forma efusiva de la PIF, asimismo hubo compromiso ocular con los depósitos queratínicos en el endotelio corneal.

\section{HALLAZGOS MICROSCOPICOS}

En este caso se observó un CGC hepático moderadamente diferenciado con intensa desmoplasia y un estroma denso de colágeno que separa las estructuras glandulares y tubulares. De igual forma los conductillos biliares neoplásicos están dispuestos en forma acinar y tubular revestidos por células epiteliales cubicas o cilíndricas anaplásicas y separados por tejido conectivo fibroso e infiltrados por células inflamatorias mononucleares (Figura 5A) y alrededor de los conductos neoplásicos infiltrados de polimorfonucleares neutrófilos y tejido necrosado (Figura 5B). De acuerdo con los siguientes hallazgos macroscópicos encontrados en el paciente producidos sobre la serosa del pulmón como la proliferación de fibroblastos que resultaron en una gruesa capa de fibrina adherida a la serosa, acompañada de congestión pulmonar alveolar difusa, causados probablemente por la activación producida por el virus causante de la PIF, en el intestino delgado una enteritis con acortamiento y destrucción de las vellosidades intestinales (Figura $5 \mathrm{C}$ ) y en el riñón una nefritis intersticial linfoplasmocitaria (Figura 5D), permite inferir un proceso viral.
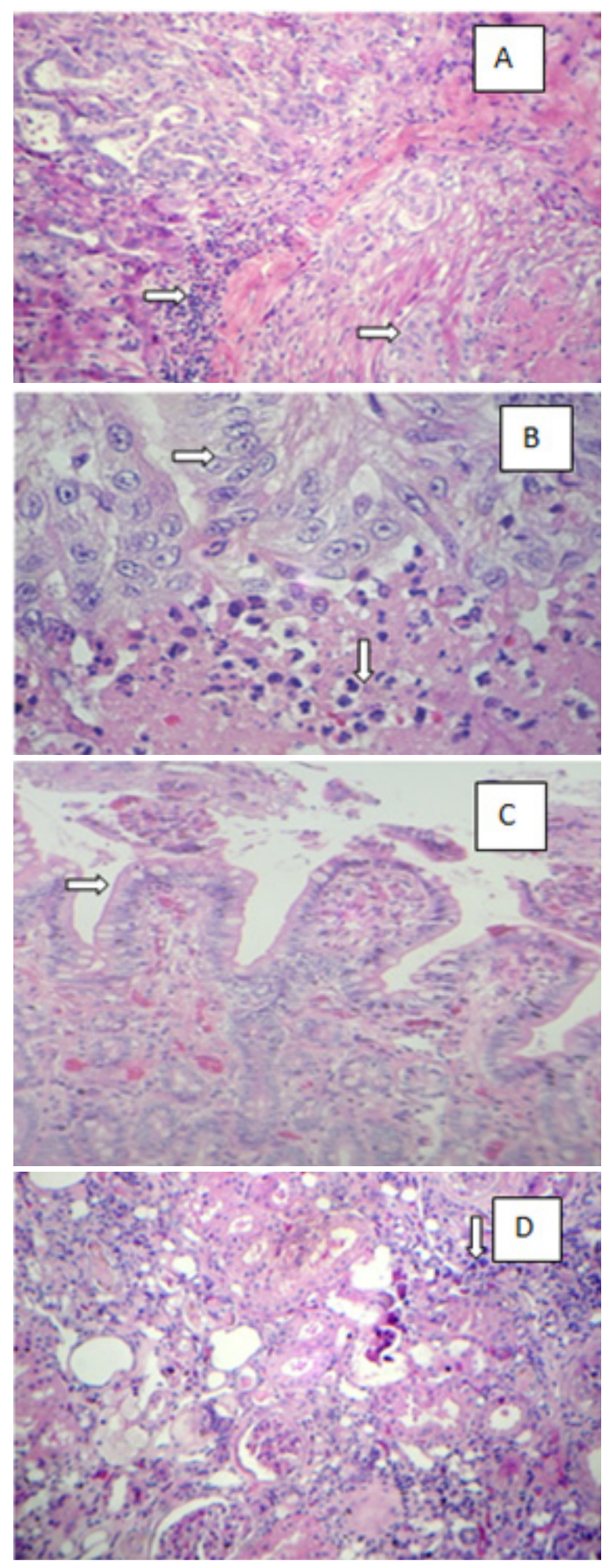

Figura 5. CGC hepático moderadamente diferenciado con estructuras glandulares y tubulares, 10X (A). Conductos neoplásicos rodeadas por infiltrados de polimorfonucleares $y$ tejido necrosado (B), 40X. Acortamiento y destrucción de las vellosidades intestinales, 10X, (C). Nefritis intersticial linfoplasmocítica, 10X (D). 


\section{DISCUSIÓN}

Aunque los resultados de los hallazgos histopatológicos son una herramienta útil para el diagnóstico de la PIF, éstos sólo pueden interpretarse en correlación con los signos clínicos $(14,15)$. De acuerdo a los signos clínicos y los resultados de las pruebas paraclínicas, linfopenia absoluta y neutrofília absoluta y relativa encontrada en el hemograma junto al exudado no séptico peritoneal coincide con Alleman (16), Lappin y Turnwald (17) quienes reportan la predominancia de neutrófilos no degenerados en el conteo total de células y niveles elevados de proteína para la PIF, por lo que el diagnóstico clínico fue orientado hacia una PIF. Morfológicamente los cambios anatomopatológicos observados como derrames abdominales, torácicos, plétora sanguínea en el epicardio e igualmente el acortamiento con destrucción de las vellosidades intestinales y una moderada inflamación de ellas, sugieren la presencia de un proceso viral concomitante con la forma efusiva de la PIF $(10,18,19)$, asimismo hubo compromiso ocular con los depósitos queratínicos en el endotelio corneal (13), algunos de los hallazgos podrían también ser ocasionados por la neoplasia hepática como las efusiones peritoneales $(17,20)$, aunque en gatos la ascitis y la hipoalbuminemia son menos comunes como consecuencia del CGC (2).

Debido a que no fue descrito microscópicamente el patrón nodular sobre la superficie visceral de estómago y del mesenterio queda impreciso determinar si éstos son focos de la PIF o son metástasis del colangiocarcinoma (17, 20). En cuanto a la descripción citológica de las células obtenidas por abdominocentesis, éstas son compatibles con las de un colangiocarcinoma, se observaron como sabanas planas o grupos de células cuboidales o células columnares bajas en forma de "panal de abeja", sus núcleos fueron coloreados oscuros y de forma redondeda a ovalada con un patrón de cromatina finamente reticulada y escaso citoplasma, correspondientes a células atípicas de los conductillos biliares (21). Igualmente, los cambios morfológicos observados en el hígado confirman el colangiocarcinoma hepático (2, 6, 22-24). Para concluir es esencial en los gatos incluir al CGC en el diagnóstico diferencial de las enfermedades abdominales no específicas y establecer el pronóstico debido a que el CGC hace metástasis (1), además de la histopatología existen nuevas alternativas de diagnóstico que permiten soportar un diagnóstico más preciso, por lo cual se ve la necesidad de implementar otras técnicas como la inmunohistoquímica usando anticuerpos monoclonales de ratón (FIPV3- 70, Custom Monoclonals, West Sacramento, USA) para determinar la presencia del antígeno del coronavirus felino, agente causante de la PIF y reportar así un diagnóstico definitivo (25), - la prueba de la reacción en cadena de la polimerasa transcriptasa reversa (RT-PCR), la que se convirtió en una herramienta valiosa para la detección de ácido nucleíco de coronavirus en la sangre, en los derrames de la cavidad,en las heces y en las muestras de tejido de los felinos infectados $(8,14)$.

En humanos la aparición de los tumores hepáticos está relacionada con varias alteraciones moleculares, siendo la más evidente la alteración de los mecanismos que regulan el equilibrio entre proliferación y muerte celular. En cuanto a las terapéuticas que actualmente se están investigando en ésta especie está VAS2870, un fármaco que frena el crecimiento de los tumores hepáticos y que está en proceso de evaluación para patologías cardiovasculares, el VAS2870, inhibe in vitro las enzimas de la familia de las NADPH oxidasas en células tumorales del hígado, induciendo la apoptosis. De ese modo, el fármaco consigue reducir el crecimiento celular y recuperar la capacidad de la célula de autodestruirse, como respuesta al factor de crecimiento de transformación beta (TGF-beta) (26).

\section{REFERENCIAS}

1. Filgueira KD, Reis PFC da C, Freitas VAL de, Paula VV de. Cholangiocarcinoma in domestic feline: a case report. Journal Medvep 2009; 7(20):113-116.

2. Cullen JM, Popp JA. Tumors on the liver and gall bladder. En: Meuten DJ. Tumors in domestic animals. 4 Ed. Iowa (USA): Iowa 2002.

3. Fatma I, ZabitY, Sevil AV, Mehmet H. Mucinous Cholangiocarcinoma with metastases in a Turkish van cat. A case report. Bull Vet Inst Pulawy 2008; 52:131-134.
4. Patnaik AK, Lieberman $\mathrm{PH}$, Erlandson $A R$, Antonescu C. Hepatobiliary Neuroendocrine Carcinoma in Cats: A Clinicopathologic, Immunohistochemical and Ultrastructural Study of 17 Cases. Vet Pathol 2005; 42:331-337.

5. Crawford JM. El Hígado y las vías biliares. En:Cotran R, Kumar V, Collins T. Robbins Patologia Estructural y Funcional. 6 ed. Mexico (Mexico): Interamericana; 2000. 
6. Cullen JM, Maclachlan. Liver, biliary system and exocrine pancreas. En: McGavin DM, Carlton WW, Zachary JF. Thomson's Special Veterinary Pathology. Missouri, USA: Mosby; 2001.

7. Dean GA, Olivry T, Stanton C, Pedersen NC. In vivo cytokine response to experimental feline infectious peritonitis virus infection. Vet Microbiol 2003; 97:1-12.

8. Benetka V, Heiss-kübber A, Kolodziejek J, Nowotny N, Parisot-Hofmann M, Möstl K. Prevalence of feline coronavirus types I and II in cats with histopathologically verified feline infectious peritonitis. Vet Microbiol 2004; 99: 31-42.

9. Hohdatsu $\mathrm{T}$, Yamato $\mathrm{H}$, Ohkawa $\mathrm{T}$, Kaneko M, Motokawa K, Kusuhara H, Kaneshima T, Arai S, Koyama H. Vaccine efficacy of a cell lysate with recombinant baculovirus expressed feline infectious peritonitis (FIP) virus nucleocapsid protein against progression of FIP. Vetn Microbiol 2003; 97: 31-44.

10. Tizard IR. Resistencia a los virus. En: Inmunología veterinaria. 6 ed. México: McGraw Hill Interamericana; 2002.

11. Kennedy M, Boedeker N, Gibbs P, Kania S. Deletions in the 7a ORF of feline coronavirus associated with an epidemic of feline infectious peritonitis. Vet Microbiol 2001; 81: 227-234.

12. Lin CN, Su BL, Wana CH, Hsieh MW, Chveh TJ, Chueh LL. Genetic diversity and correlation with feline infectious peritonitis of feline coronavirus type I and II: A 5-year study in taiwan. Vet Microbiol 2009; 136: 233-239.

13. Addie D, Belák $S$, Boucraut-Baralon C, Egberink H, Frymus T, Gruffydd-Jones T, et al. Feline Infectious Peritonitis. J Feline Med Surg 2009; 11:594-604.

14. Simons FA, Vennema $H$, Rofina JE, Pol JM, Horzinek MC, Rottier PJM, Egberink HF. A Mrna PCR for the diagnosis of feline infectious peritonitis. J Virol Methods 2005; 124:111-116.

15. Poder SL. Péritonite infectieuse féline. EMCVétérinaire 2005; 2:169-178.

16. Alleman AR. Abdominal, thoracic, and pericardial effusions. Vet Clin Small Anim 2003; 33(1):92-105.
17. Lappin MR, Turnwald GH. Microbiology and Infectious Disease. En: Willard $M$, Tvedten $H$ (Eds). Small animal clinical diagnosis by laboratory methods. 4 ed. (USA): Saunders. Elsevier; 2004. p 350-351.

18. Petter CD. Relação entre albumina e globulina na efusão de gatos suspeitos de peritonite infecciosa felina (PIF). [Monografia de especialização em Análises Clínicas Veterinárias]. Porto Alegre: Universidade Federal do Rio Grande do Sul, Faculdade de Veterinária; 2007.

19. Rottier PJ, Nakamura $K$, Schellen P, Volders $\mathrm{H}$, Haijema, BJ .Acquisition of macrophage tropism during the pathogenesis of feline infectious peritonitis is determined by mutations in the feline coronavirus spike protein. J Virol 2005; 79(22):14122-30.

20. Latimer $\mathrm{K}$, Prasse KW. Leukocytes. En: Latimer K, Mahaffey EA, Prasse KW. Veterinary Laboratory Medicine. Clinical Pathology. 4 ed. Iowa (USA): Blackwell Publishing; 2003.

21. Weiss DJ, Moritz A. Liver citology. Vet Clin Small Anim 2002; 32(1):1270.

22. Teredesai A, Wo“hrmann T, Schlage W. Hepatocholangiocellular Carcinoma in a Rat - Case Report. J Vet Med 2002; 49:541-544.

23. Vale $O E$, Madrigal $K$, Admadé $M$, Vale OR, Moreno A, Simoes D. Peritonitis Infecciosa Felina, Gastroenteritis y Colangiohepatitis parasitaria (Platinosomiasis) con Colangiocarcinoma Hepático: Estudio Clínico y Anatomopatológico de tres casos. Rev Cient (Maracaibo). 2005; 15(3):195-203.

24. Rosciani AS, Merlo WA, Insfrán RM, Benítez JS, Locket M, Koscinczuk P. Caracterización citológica de hepatopatías en perros y gatos. Citología en hepatopatías. Rev Vet 2007; 18(2):111-116.

25. Declercq J, De Bosschere H, Schwarzkopf LI, Declercq L. Papular cutaneous lesions in a cat associated with feline infectious peritonitis. Journal Compilation 2008; 19:255-258.

26. Sancho P, Fabregat I. The NADPH oxidase inhibitor VAS2870 impairs cell growth and enhances TGF- $\beta$-induced apoptosis of liver tumor cells. Biochem Pharmacol 2011, 81(7):917-924. 